\title{
Loss of nuclear p27 (CDKN1B/KIP1) in colorectal cancer is correlated with microsatellite instability and CIMP
}

Shuji Ogino ${ }^{1,2,3}$, Takako Kawasaki², Gregory J Kirkner ${ }^{4}$, Taiki Yamaji ${ }^{5}$, Massimo Loda ${ }^{1,2,3}$ and Charles S Fuchs $s^{2,3,4}$

${ }^{1}$ Department of Pathology, Brigham and Women's Hospital, Boston, MA, USA; ${ }^{2}$ Department of Medical Oncology, Dana-Farber Cancer Institute, Boston, MA, USA; ${ }^{3}$ Harvard Medical School, Boston, MA, USA; ${ }^{4}$ Department of Medicine, Brigham and Women's Hospital, Boston, MA, USA and ${ }^{5}$ Department of Epidemiology, Harvard School of Public Health, Boston, MA, USA

\begin{abstract}
Downregulation of p27 (cyclin-dependent kinase inhibitor-1B, CDKN1B or KIP1) is caused by increased ubiquitin-mediated proteasomal degradation in colorectal cancer, and has been associated with poor prognosis. CpG island methylator phenotype (CIMP) is a phenotype of colorectal cancer with extensive promoter methylation, and associated with high degree of microsatellite instability (MSI-H) and BRAF mutations. We have recently shown that both CIMP and MSI-H are inversely associated with downregulation of p21 (CDKN1A or CIP1), another cyclin-dependent kinase inhibitor. However, no study to date has examined relationship between p27 and CIMP status in colorectal cancer. Using MethyLight assays, we measured DNA methylation in five CIMP-specific gene promoters \{CACNA1G, CDKN2A (p16), CRABP1, MLH1 and NEUROG1\} in 706 colorectal cancer samples obtained from two large prospective cohorts. Among the 706 tumors, 112 (16\%) were CIMP-high tumors with $\geq 4 / 5$ methylated promoters. We assessed p27 and p53 expressions by immunohistochemistry. Loss of nuclear p27 expression \{observed in 231 tumors (33\%)\} was significantly associated with CIMP-high, MSI-H and BRAF mutations, and these associations were much more pronounced among p53-negative tumors than p53-positive tumors. When CIMP-high and non-CIMP-high tumors were stratified by MSI status (or KRAS and BRAF status), CIMP-high and MSI-H (but not BRAF mutations) were still significantly associated with nuclear p27 loss. Nuclear p27 loss did not appear to be directly related to CDKN2A (p16) methylation. We conclude that downregulation of nuclear p27 is associated with CIMP-high and MSI-H in colorectal cancer. These associations are stronger among p53 wild-type tumors, implying important interplay of p27 and p53 functions (or dysfunctions) in the development of various molecular subtypes of colorectal cancer. Modern Pathology (2007) 20, 15-22. doi:10.1038/modpathol.3800709; published online 3 November 2006
\end{abstract}

Keywords: colon cancer; CIMP; p27; p53; cyclin-dependent kinase inhibitor; MSI

Progression through the cell cycle involves sequential activation and inactivation of cyclin-dependent kinases (CDKs). ${ }^{1}$ CDKs are activated through association with positive regulators (cyclins) and inactivated by cyclin-CDK inhibitors. p27 (cyclindependent kinase inhibitor 1B, CDKN1B or KIP1) is one of the cyclin-CDK inhibitors and plays a key role in preventing progression into S-phase of the cell cycle. ${ }^{1}$ Regulation of p27 levels is achieved post-translationally through ubiquitin-mediated

Correspondence: Dr S Ogino, MD, PhD, Department of Pathology, Brigham and Women's Hospital, Harvard Medical School, 75 Francis St., Boston, MA 02115, USA.

E-mail: shuji_ogino@dfci.harvard.edu

Received 25 July 2006; revised 25 August 2006; accepted 28

August 2006; published online 3 November 2006 protein degradation. ${ }^{2}$ The F-box protein SKP2 has been identified as the substrate recognition component that binds and targets p27 for ubiquitination and subsequent degradation. ${ }^{3,4}$ Low levels of p27 have been associated with tumor progression and poor prognosis in various cancers including colon, breast and prostate cancers. ${ }^{5-8}$ Although mutations or deletions of p27 allele rarely occur, downregulation of p27 in colorectal cancer mainly result from abnormal activation of ubiquitin-mediated proteolysis. ${ }^{5,6} C D K N 1 B$ (p27) promoter has not been shown to be significantly methylated in colorectal cancer. ${ }^{9}$

Transcriptional inactivation by cytosine methylation at promoter $\mathrm{CpG}$ islands of tumor suppressor genes is thought to be an important mechanism in human carcinogenesis. ${ }^{10,11} \mathrm{~A}$ number of tumor suppressor genes, including CDKN2A (p16/INK4A), 
MGMT, MLH1, etc., are silenced by promoter methylation in colorectal cancer. ${ }^{10,11}$ A subset of colorectal cancers exhibit promoter methylation in multiple genes, referred to as the $\mathrm{CpG}$ island methylator phenotype (CIMP). ${ }^{10,12,13}$ CIMP-positive colorectal tumors appear to have a distinct clinical, pathologic and molecular profile, including associations with female sex, proximal tumor location, mucinous and poor differentiations, microsatellite instability (MSI) and BRAF mutations. ${ }^{12,14-20} \mathrm{~A}$ recent report suggests a possible association between JC virus and CIMP in colorectal cancer. ${ }^{21}$ We have recently demonstrated that both MSI and CIMP are inversely associated with downregulation of p21 (CDKN1B/KIP1), another cyclin-dependent kinase inhibitor. ${ }^{22}$ However, no study to date has examined relationship between p27 expression and CIMP. In addition, previous studies have not shown any correlation for p27 expression with MSI status ${ }^{23}$ or mutations in the TGFBR2 gene (encoding TGF- $\beta$ receptor type II) within MSI-H tumors. ${ }^{24}$ In this study, using quantitative real-time PCR (MethyLight) assays, ${ }^{19,25-27}$ and population-based samples of colorectal cancer from two large prospective cohort studies, we have shown correlations of loss of nuclear p27 expression with CIMP and MSI. MethyLight assays can reliably distinguish high from low levels of DNA methylation, the latter of which likely have little or no biological significance. ${ }^{28}$

\section{Materials and methods}

\section{Study Group}

We utilized the databases of two large prospective cohort studies; the Nurses' Health Study $\left(N=121700\right.$ women followed since 1976) ${ }^{29}$ and the Health Professional Follow-up Study $(N=51500$ men followed since 1986). ${ }^{30}$ Informed consent was obtained from all participants before inclusion in the cohorts. All cohort participants were free of cancer (except for nonmelanoma skin cancer) at the time of study entry. A subset of the cohort participants developed colorectal cancers during prospective follow-up. We included cases only if there was adequate paraffin-embedded tumor tissue and results were available for DNA methylation and p27 at the time of this study. As a result, a total of 706 colorectal cancer cases (316 from the men's cohort and 390 from the women's cohort) were included in this study. Most cases have been previously characterized for status of CIMP, MSI, KRAS, BRAF and p53. ${ }^{19,22,31}$ However, no tumor has previously been studied for $\mathrm{p} 27$. Tissue collection and analyses were approved by the Institutional Review Boards.

\section{MSI Analysis}

Genomic DNA was extracted as previously described. ${ }^{32}$ Whole genome amplification (WGA) of genomic DNA was performed by PCR using random 15-mer primers. ${ }^{33}$ Methods for MSI analysis were previously described. ${ }^{34}$ In addition to D2S123, D5S346, D17S250, BAT25 and BAT26 (the NCI panel), we used BAT40, D18S55, D18S56, D18S67 and D18S487 (ie, a 10-marker panel). 'MSI-high (MSI-H)' was defined as instability in $30 \%$ or more of the markers, 'MSI-low (MSI-L)' as instability in $<30 \%$ of the markers, and 'microsatellite stability (MSS)' as no unstable marker.

\section{Quantitative Real-Time PCR for DNA Methylation (MethyLight)}

Sodium bisulfite treatment on genomic DNA was performed as previously described. ${ }^{28}$ Real-time PCR to measure DNA methylation (MethyLight) was performed as previously described. ${ }^{25-27}$ We used ABI 7300 (Applied Biosystems, Foster City, CA, USA) for quantitative real-time PCR on five CIMPspecific promoters (CACNA1G, CDKN2A (p16/ INK4A), CRABP1, MLH1 and NEUROG1). ${ }^{19}$ We have previously validated the selection and use of these five markers as a CIMP-specific promoter panel. ${ }^{19}$ COL2A1 (the collagen 2A1 gene) was used to normalize for the amount of input bisulfite-converted DNA. ${ }^{27,28}$ Primers and probes were previously described: CACNA1G, CRABP1 and NEUROG1;19 CDKN2A and COL2A1; ${ }^{27}$ and MLH1 ${ }^{28}$ Percentage of methylated reference (PMR) was determined in each locus, and methylation positivity was defined as PMR $\geq 4$ (or $\geq 6$ for $C R A B P 1$ ) as previously validated. ${ }^{26-28,35}$ Precision and performance characteristics of bisulfite conversion and subsequent MethyLight assays have been previously evaluated and the assays have been validated. ${ }^{28}$

The CpG island methylator phenotype-high (CIMP-high) was defined as the presence of $\geq 4 / 5$ methylated promoters. ${ }^{19}$ CIMP-low was defined as the presence of 1/5-3/5 methylated promoters, and CIMP-0 was defined as the absence of methylation in any of the five promoters. ${ }^{36}$

\section{Sequencing of KRAS and BRAF}

PCR and sequencing targeted for KRAS codons 12 and 13 and $B R A F$ codon 600 have been performed as previously described. ${ }^{33,37}$

\section{TMA and Immunohistochemistry for p27 and p53}

Tissue microarrays (TMAs) were constructed for p53 analysis as previously described, ${ }^{31}$ using the Automated Arrayer (Beecher Instruments, Sun Prairie, WI, USA).

Methods for p27 immunohistochemistry were previously described. ${ }^{37}$ The extent of nuclear p27 expression was visually estimated using whole tissue sections, and interpreted as 'loss' (no staining, 
only weakly staining, or $<10 \%$ of tumor cells positive for moderate/strong staining) (Figure 1), moderate/strong positive in $20-49 \%$ of cells $(1+)$, or moderate/strong positive in $\geq 50 \%$ of cells $(2+)$ (Figure 2). Inflammatory cells and normal colonic epithelial cells served as internal positive controls.

For p53 immunohistochemistry, deparaffinized tissue sections in a citrate buffer (BioGenex, San Ramon, CA, USA) were microwaved in a pressure cooker at high power for $15 \mathrm{~min}$. Tissue sections were incubated with $3 \% \mathrm{H}_{2} \mathrm{O}_{2}(10 \mathrm{~min})$ to block endogenous peroxidase, and then incubated with protein block (Vector Laboratories, Burlingame, CA, USA) (10 min). Primary anti-p53 mouse monoclonal antibody (clone DO-1, Calbiochem, San Diego, CA, USA) (dilution 1:50) was applied for $30 \mathrm{~min}$ at room temperature. Then, biotinylated secondary MultiLink antibody (Biogenex) was applied (20 min),

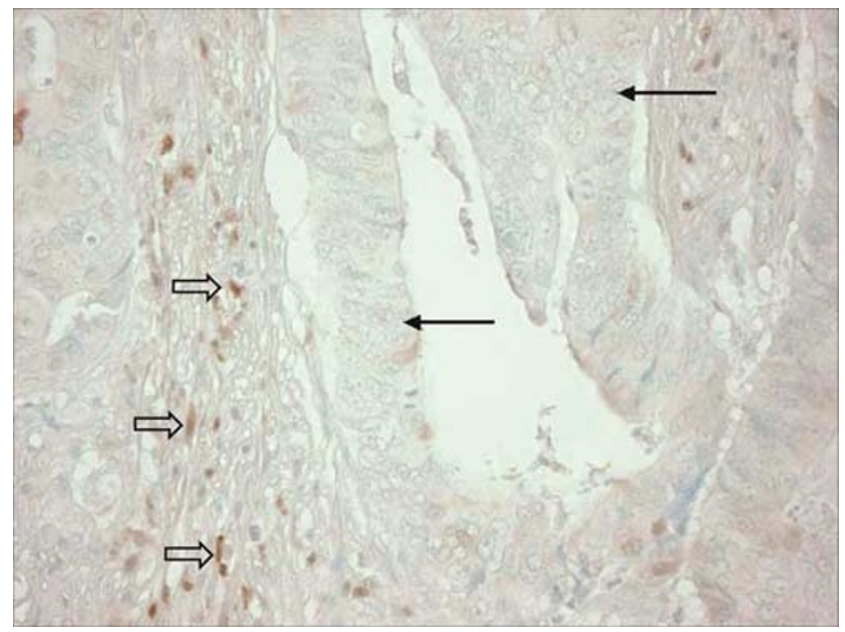

Figure 1 Loss of Nuclear p27 in Colorectal Cancer. Tumor cells with loss of p27 expression are indicated by arrows. Normal mesenchymal cells and inflammatory cells serve as internal positive controls (empty arrows).

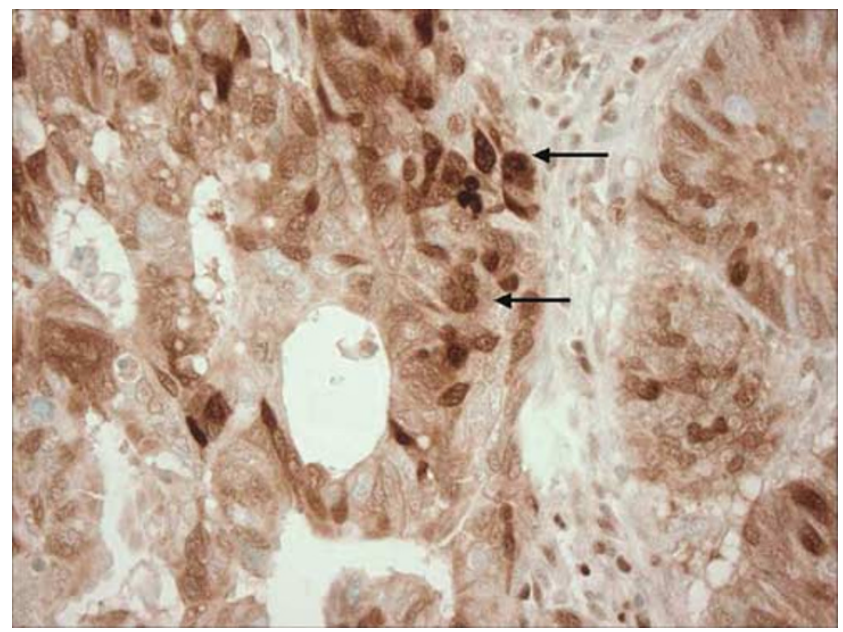

Figure 2 Colorectal Cancer Cells with Nuclear p27 Expression. Tumor cells with nuclear p27 expression are indicated by arrows. horse radish peroxidase avidin complex (Biogenex) was added $(20 \mathrm{~min})$ and sections were visualized by $\mathrm{DAB}$ (5 min) and methyl-green counterstain. We visually estimated the fraction of tumor cells with strong and unequivocal nuclear staining for p53, by examining at least two tissue cores in TMAs, or the whole tissue section in each case for which there was not enough tissue for TMAs or results were equivocal in TMAs. p53 positivity was defined as $50 \%$ or more of tumor cells with unequivocal strong nuclear staining.

Appropriate positive and negative controls were included in each run of p27 and p53 immunohistochemistry. All immunohistochemically stained slides were interpreted by a pathologist (SO) blinded from any other laboratory data.

\section{Statistical Analysis}

In statistical analysis, $\chi^{2}$ test (or Fisher's exact test when the number of cases in any category was $<10$ ) was performed for categorical data, using the SAS program (version 9.1, SAS Institute, Cary, NC, USA). All $P$-values were two-sided, and statistical significance was set at $P \leq 0.05$.

\section{Results}

\section{Loss of p27 is Correlated with MSI and CIMP}

We examined p27 expression in 706 colorectal cancer specimens by immunohistochemistry, with 231 tumors (33\%) showing p27 loss. We also quantified DNA methylation in a panel of the five CIMP-specific promoters (CACNA1G, CDKN2A, CRABP1, MLH1 and NEUROG1) ${ }^{19}$ by MethyLight, with 112 tumors (16\%) classified as CIMP-high ( $\geq 4 / 5$ methylated promoters). We have previously validated the selection and use of these five markers for the determination of CIMP status. ${ }^{19}$

Colorectal cancers with p27 loss showed a significantly higher frequency of CIMP-high $(27 \%)$ than p27 $1+$ tumors $(7.0 \%, P<0.0001)$ and than p27 $2+$ tumors $(16 \%, P=0.02)$ (Table 1$)$. With regard to p27 and MSI status, colorectal cancers with p27 loss showed a significantly higher frequency of MSI-H $(26 \%=60 / 231)$ than p27 $1+$ tumors $(9.9 \%=28 / 283, P<0.0001)$ and p27 $2+$ tumors $(14 \%=17 / 120, P=0.01)$.

In order to determine whether the association of p27 loss with CIMP-high was due to methylation of CDKN2A (p16, another important cell cycle regulator) rather than CIMP-high, we examined the frequencies of p27 loss among CIMP-high and CIMP-low/0 tumors stratified by $C D K N 2 A$ methylation status (Supplementary Material). For this analysis, CDKN2A was excluded from the CIMP panel to avoid its confounding effect on diagnosing CIMP status, and CIMP-high was defined as the presence of $\geq 3 / 4$ methylated promoters. The fre- 
quencies of p27 loss were higher among CIMP-high tumors than CIMP-low/0 even after tumors were stratified by $C D K N 2 A$ methylation status. In contrast, after stratification by CIMP status, CDKN2Amethylated and unmethylated tumors did not show a significant difference in the frequencies of p27 loss. Therefore, p27 loss was associated primarily with CIMP-high, rather than CDKN2A methylation.

\section{Combined p27/p53 Status, CIMP and MSI}

As a result of important roles of both p27 and p53 in regulating the cell cycle, we correlated combined status of p27 and p53 with CIMP and MSI. The association of p27 loss with CIMP-high or MSI-H was much more pronounced among p53-negative tumors than p53-positive tumors (Table 2). For instance, p53-negative/p27-loss tumors showed significantly higher frequencies of CIMP-high and MSI$\mathrm{H}$ than p53-negative/p27 $1+$ tumors and than p53negative/p27 $2+$ tumors (Table 2). In contrast, there was no significant difference in CIMP-high or MSI-H frequencies among p53-positive tumor groups.

\section{Combined p27/p53 Status, and KRAS/BRAF Mutations}

We correlated combined status of p27 and p53 with KRAS and BRAF mutations (Table 3). p53-negative/ p27-loss tumors showed a significantly higher frequency of BRAF mutations (28\%) than p53negative/p27 $1+$ tumors $(8.9 \%, P=0.0003)$ and p53-negative/p27 $2+$ tumors $(15 \%, P=0.04)$ (Table $3)$. In contrast, there was no significant difference in the frequencies of BRAF mutations among p53positive tumor groups.

Table 1 Frequencies of CIMP-0, CIMP-low and CIMP-high in colorectal cancers with various p27 status

\begin{tabular}{|c|c|c|c|c|c|c|c|c|c|c|}
\hline & \multicolumn{6}{|c|}{ Number of methylated promoters } & \multirow{2}{*}{$\begin{array}{l}\text { CIMP-low } \\
1 \text { to } 3\end{array}$} & \multirow{2}{*}{$\begin{array}{l}\text { CIMP-high } \\
\quad \geq 4\end{array}$} & & \\
\hline & $O(C I M P-0)$ & 1 & 2 & 3 & 4 & 5 & & & & \\
\hline All cases $(N=706)$ & $360(51 \%)$ & 117 & 75 & 42 & 44 & 68 & $234(33 \%)$ & $112(16 \%)$ & \multirow{4}{*}{$\begin{array}{l}P<0.0001 \\
P=0.004\end{array}$} & \multirow{4}{*}{$P=0.02$} \\
\hline p27 loss $(N=231)$ & $92(40 \%)$ & 36 & 23 & 18 & 23 & 39 & $77(33 \%)$ & $62(27 \%)$ & & \\
\hline $\begin{array}{l}\text { p27 } 1+\text { (expressed in } 20 \% \text { to } \\
49 \% \text { of cells })(N=284)\end{array}$ & $166(58 \%)$ & 49 & 36 & 13 & 12 & 8 & $98(35 \%)$ & $20(7.0 \%)$ & & \\
\hline p27 2+ (expressed in $\geq 50 \%$ & $76(62 \%)$ & 14 & 7 & 6 & 5 & 15 & $27(22 \%)$ & $20(16 \%)$ & & \\
\hline
\end{tabular}

of cells $)(N=123)$

CIMP, CpG island methylator phenotype.

Important values are in bold.

Table 2 Frequencies of CIMP-high and MSI-H in colorectal cancers with various combined p53/p27 status

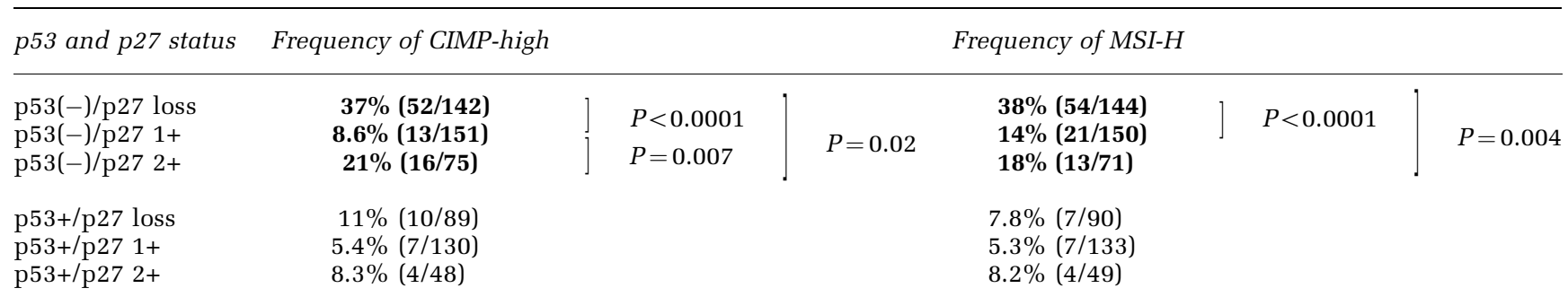

CIMP, CpG island methylator phenotype; MSI-H, microsatellite instability-high.

Important values are in bold.

Table 3 Frequencies of $K R A S$ and BRAF mutations in colorectal cancers with various combined p53/p27 status

\begin{tabular}{|c|c|c|c|c|c|}
\hline p53 and p27 status & KRAS mutation frequency & & BRAF mutation frequency & & \\
\hline $\begin{array}{l}\mathrm{p} 53(-) / \mathrm{p} 27 \text { loss }(N=140) \\
\text { p53(-)/p27 } 1+(N=146) \\
\text { p53(-)/p27 } 2+(N=68)\end{array}$ & $\begin{array}{l}29 \%(40) \\
36 \%(53) \\
44 \%(30)\end{array}$ & $P=0.03$ & $\begin{array}{r}28 \%(39) \\
8.9 \%(13) \\
15 \%(10)\end{array}$ & $P=0.0003$ & $P=0.04$ \\
\hline $\begin{array}{l}\text { p53+/p27 loss }(N=89) \\
\text { p53+/p27 } 1+(N=128) \\
\text { p53+/p27 } 2+(N=47)\end{array}$ & $\begin{array}{l}37 \%(33) \\
28 \%(36) \\
36 \%(17)\end{array}$ & & $\begin{array}{l}15 \%(13) \\
6.3 \%(8) \\
6.4 \%(3)\end{array}$ & & \\
\hline
\end{tabular}

Important values are in bold. 
p27 Loss in Various MSI/CIMP Subtypes of Colorectal Cancer

In order to examine an association between p27 loss and CIMP-high (or MSI-H) independent of MSI (or CIMP) status, we examined the frequencies of p27 loss in six subtypes of colorectal cancer according to combined status of MSI and CIMP (Figure 3). Among MSI-H tumors, MSI-H CIMP-high tumors showed a significantly higher frequency of p27 loss $(62 \%=48 / 78)$ than MSI-H CIMP-low tumors $(17 \%=2 / 12, P=0.005)$ (Figure 3). Among MSI-L/ MSS tumors, MSI-L/MSS CIMP-high tumors showed a significantly higher frequency of p27 loss $(41 \%=14 / 34)$ than MSI-L/MSS CIMP-0 tumors (24\% $=79 / 330, P=0.03)$. Thus, p27 loss appeared to be associated with CIMP-high after stratification by MSI status.

We also stratified tumors by CIMP status. Among CIMP-high tumors, MSI-H CIMP-high tumors showed a higher frequency of p27 loss (62\%) than MSI-L/MSS CIMP-high tumors $(41 \%, P=0.05)$. Among CIMP-0 tumors, MSI-H CIMP-0 tumors showed a higher frequency of p27 loss $(48 \%=$

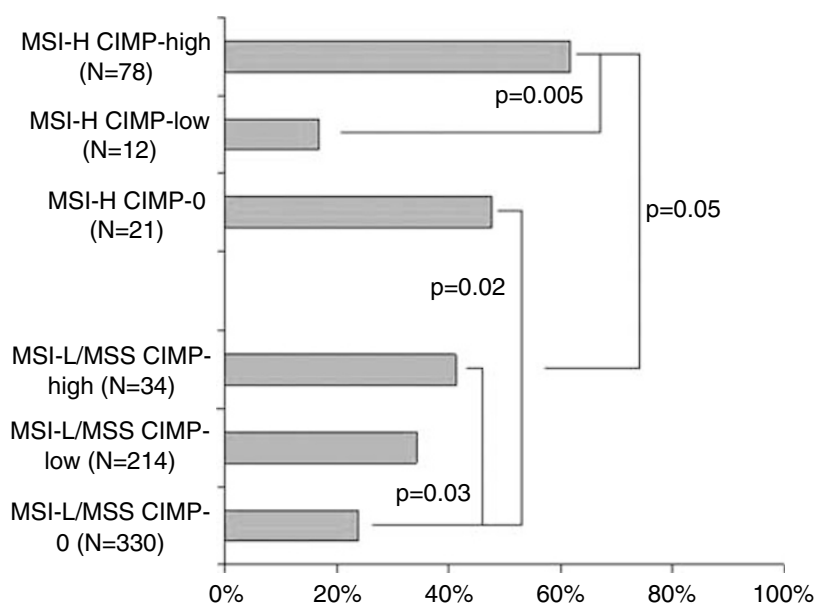

Figure 3 Frequency of p27 Loss in Various MSI/CIMP Subtypes of Colorectal Cancer. p27 loss is associated with CIMP-high (or MSI$\mathrm{H}$ ) independent of MSI status (or CIMP status). CIMP, CpG island methylator phenotype; MSI, microsatellite instability; MSS, microsatellite stable.

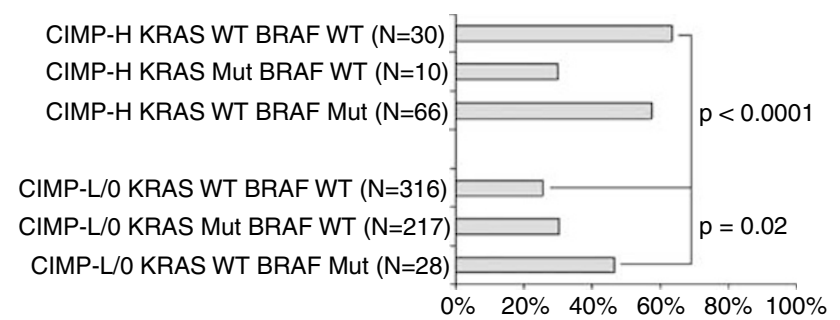

Figure 4 Frequency of p27 Loss in Various CIMP/KRAS/BRAF Subtypes of Colorectal Cancer. p27 loss appears to be associated with CIMP-high independent of KRAS/BRAF status. CIMP-H, CpG island methylator phenotype-high; CIMP-L/0, CIMP-low/0; Mut, mutant; WT, wild-type.
10/21) than MSI-L/MSS CIMP-0 tumors (24\%, $P=0.02)$. Therefore, p27 loss appeared to be associated with MSI-H independent of CIMP status.

\section{p27 Loss in Various CIMP/KRAS/BRAF Subtypes of Colorectal Cancer}

In order to examine an association between p27 loss and KRAS/BRAF mutations independent of CIMP status, we examined the frequencies of p27 loss in six subtypes of colorectal cancer according to combined status of CIMP/KRAS/BRAF (Figure 4). Among CIMP-low/0 tumors, CIMP-low/0 BRAFmutated tumors showed a higher frequency of p27 loss $(46 \%=13 / 28)$ than CIMP-low/0 KRAS/BRAF wild-type tumors $(26 \%=83 / 316, P=0.02)$ (Figure 4). In particular, among CIMP-low tumors, p27 loss was significantly more common in CIMP-low BRAFmutated (KRAS wild-type) tumors $(55 \%=11 / 20)$ than CIMP-low KRAS/BRAF wild-type tumors $(30 \%=29 / 98, P=0.04)$. However, no association between p27 loss and BRAF mutations was observed among CIMP-high and CIMP-0 tumors. Thus, p27 loss was associated with BRAF mutations only among CIMP-low tumors.

\section{Discussion}

We conducted this study to evaluate relationship between downregulation of p27 and well-characterized molecular features of colorectal cancer, including CIMP, MSI and mutations in the KRAS and $B R A F$ oncogenes. We have demonstrated that there were positive correlations for loss of nuclear p27 expression with CIMP-high and MSI-high (MSI-H) in colorectal cancer. Our results are in contrast to a study by Edmonston et a ${ }^{23}$ who found no association between p27 loss and MSI. In addition, we have shown that p27 $1+$ tumors showed lower frequencies of CIMP-high, MSI-H and BRAF mutations, compared to p27-lost tumors and p27 $2+$ tumors. Further studies are necessary to determine whether p27 $1+$ tumors are biologically distinct from p27 $2+$ tumors.

As CIMP-high and MSI-H (as well as CIMP-high and BRAF mutations) are tightly associated with each other, ${ }^{16-19}$ we stratified tumors according to CIMP and MSI status, and according to CIMP and $K R A S / B R A F$ gene status. The association of p27 loss with CIMP-high (or MSI-H) appeared to be independent of MSI (or CIMP) status. In addition, the association of p27 loss with CIMP-high also appeared to be independent of $B R A F$ status. However, the association of p27 loss with BRAF mutations appeared to be secondary to the associations between BRAF mutations and CIMP-high and between CIMP-high and p27 loss. It is conceivable that a small number of CIMP-high tumors might have been classified as CIMP-low by our strict criteria for CIMP-high (ie, $\geq 4 / 5$ methylated promo- 
ters), although we have validated our CIMP-high criteria and tumors with $1 / 5-3 / 5$ methylated promoters have a much higher KRAS mutation rate than tumors with $\geq 4 / 5$ methylated promoters. ${ }^{19,36}$ Therefore, we conclude that p27 loss is associated primarily with CIMP-high and MSI-H, but not with $B R A F$ mutations.

We utilized quantitative real-time PCR (MethyLight) to distinguish high from low levels of DNA methylation. Assays to measure DNA methylation may be potentially very useful in clinical practice. ${ }^{10}$ DNA methylation may be a useful marker for predicting prognosis and monitoring efficacy of adjuvant therapy in cancer patients ${ }^{38}$ and for risk assessment in surveillance of high- or low-risk individuals. ${ }^{39,40}$ In the current study, the use of quantitative DNA methylation assays as well as relatively unbiased, population-based samples of colorectal cancer from two large prospective cohorts has enabled us to precisely estimate the frequency of colorectal cancers with specific molecular features (such as CIMP-high, MSI-H, p27 loss, etc.) at a population level.

As a result of important roles of both p27 and p53 in regulating the cell cycle, we examined whether p53 status in colorectal cancer might modify molecular characteristics of p27-lost or p27-expressed tumors. Interestingly, the associations of p27 loss with CIMP-high, MSI-H and BRAF mutations were much more pronounced among p53negative (presumably wild-type) tumors than p53positive (presumably mutated) tumors. Our results imply that functional status of p27 may be much more important in p53 wild-type tumors than p53mutated tumors. Although p53 immunohistochemistry has been shown to have both false positives and false negatives for the assessment of TP53 gene mutations, when higher threshold of p53 positivity is used (as in the current study), p53 immunohistochemistry can generally predict the presence or absence of mutations of the TP53 gene. ${ }^{41-43}$

We have recently demonstrated inverse associations of CIMP-high and MSI-H with downregulation of p21 (CDKN1A/CIP1), another important cyclindependent kinase inhibitor. ${ }^{22}$ Taken together with our current results, CIMP-high MSI-H tumors tend to downregulate p27, whereas non-CIMP non-MSI tumors tend to downregulate p21. Further studies are necessary to elucidate exact pathogenetic links between CIMP/MSI and p27 downregulation, and between non-CIMP/non-MSI and p21 downregulation.

Our data may have significant clinical implications because of emerging importance of both DNA methylation and the ubiquitin-proteasome pathway as attractive chemotherapeutic/chemopreventive targets. ${ }^{6,10}$ The mechanisms of p27 regulation are shown to be at the post-translational level and, in particular, the ubiquitin-proteasome pathway plays an important role in downregulation of p27 in aggressive colorectal cancer., ${ }^{5,8}$ Our data might suggest that targeting both epigenetic alterations and the ubiquitin pathway might be effective against tumors with CIMP-high and/or MSI-H. In addition, for therapeutic approaches targeting p27 and/or the ubiquitin pathway, it is likely important to consider the effects of mutant or wild-type p53, potentially modulating the signaling pathway downstream of p27. Elucidating the molecular link between CIMP and p27 downregulation may be important for the purpose of developing molecularly targeted treatment against CIMP-high colorectal cancer. In addition, elucidating mechanisms that may overcome the tumor suppressor function of p27 in CIMP-low/0 tumors may also be important for the development of targeted therapy against CIMP-low/0 tumors.

The prognostic significance of p27 loss has been examined in previous studies, which have shown that p27 loss was a significant predictor of worse survival by multivariate analysis..$^{5,44,45}$ Manne et $a l^{46}$ examined p27 expression in colorectal cancer stratified by stage and found that p27 loss was associated with local recurrence and poor survival only for stage III colorectal cancer. The authors also found that p27 loss was associated with poor differentiation (in stage II tumors) and distal location. ${ }^{46}$ In contrast, Palmqvist et al ${ }^{44}$ showed that p27 loss was associated with proximal location. These discrepant results may be attributable to differences in patient populations and criteria of p27 interpretation. The prognostic significance of CIMP or MSI stratified by the status of p27 has not been studied. Our prospective cohort studies, the Nurses' Health Study $(N=120000 \text {, followed since } 1976)^{29}$ and the Health Professional Follow-up Study $(N=51000$, followed since 1986), ${ }^{30}$ are currently ongoing, and thus, relational data on patient survival and CIMP or MSI status stratified by p27 status will be available in the future.

In conclusion, loss of nuclear p27 in colorectal cancer is associated with CIMP-high and MSI-H, and these associations are much more pronounced in p53 wild-type tumors. Our results imply that there is interplay of p27 and p53 function (or dysfunction) in the development of various molecular subtypes of colorectal cancer. Exact biological significance of p27 loss and effects of p53, CIMP and MSI status need further investigations.

\section{Acknowledgements}

This work was supported by National Institute of Health (NIH) grants P01 CA87969-03 and P01 CA55075-13. We deeply thank the Nurses' Health Study and Health Professionals Follow-up Study cohort participants who have generously agreed to provide us with biological specimens and information through responses to questionnaires. We thank Graham Colditz, Walter Willett, and many other staff members who implemented and have maintained the cohort studies. We deeply thank Peter Laird, 
Daniel Weisenberger, and Mihaela Campan for assisting in the development of the MethyLight assay. No conflict of interest is present.

\section{References}

1 Macaluso M, Montanari M, Cinti C, et al. Modulation of cell cycle components by epigenetic and genetic events. Semin Oncol 2005;32:452-457.

2 Pagano M, Tam SW, Theodoras AM, et al. Role of the ubiquitin-proteasome pathway in regulating abundance of the cyclin-dependent kinase inhibitor p27. Science 1995;269:682-685.

3 Carrano AC, Eytan E, Hershko A, et al. SKP2 is required for ubiquitin-mediated degradation of the CDK inhibitor p27. Nat Cell Biol 1999;1:193-199.

4 Tsvetkov LM, Yeh KH, Lee SJ, et al. p27(Kip1) ubiquitination and degradation is regulated by the SCF(Skp2) complex through phosphorylated Thr187 in p27. Curr Biol 1999;9:661-664.

5 Loda M, Cukor B, Tam SW, et al. Increased proteasomedependent degradation of the cyclin-dependent kinase inhibitor p27 in aggressive colorectal carcinomas. Nat Med 1997;3:231-234.

6 Mani A, Gelmann EP. The ubiquitin-proteasome pathway and its role in cancer. J Clin Oncol 2005;23: 4776-4789.

7 Chetty R. p27 Protein and cancers of the gastrointestinal tract and liver: an overview. J Clin Gastroenterol 2003;37:23-27.

8 Thomas GV, Szigeti K, Murphy M, et al. Downregulation of p27 is associated with development of colorectal adenocarcinoma metastases. Am J Pathol 1998;153:681-687.

$9 \mathrm{Xu}$ XL, Yu J, Zhang HY, et al. Methylation profile of the promoter CpG islands of 31 genes that may contribute to colorectal carcinogenesis. World J Gastroenterol 2004;10:3441-3454.

10 Issa JP. CpG island methylator phenotype in cancer. Nat Rev Cancer 2004;4:988-993.

11 Laird PW. Cancer epigenetics. Hum Mol Genet 2005;14(Spec No 1):R65-R76.

12 Toyota M, Ohe-Toyota M, Ahuja N, et al. Distinct genetic profiles in colorectal tumors with or without the CpG island methylator phenotype. Proc Natl Acad Sci USA 2000;97:710-715.

13 Toyota M, Ahuja N, Ohe-Toyota M, et al. CpG island methylator phenotype in colorectal cancer. Proc Natl Acad Sci USA 1999;96:8681-8686.

14 van Rijnsoever M, Grieu F, Elsaleh H, et al. Characterisation of colorectal cancers showing hypermethylation at multiple CpG islands. Gut 2002;51:797-802.

15 Hawkins N, Norrie M, Cheong K, et al. CpG island methylation in sporadic colorectal cancers and its relationship to microsatellite instability. Gastroenterology 2002;122:1376-1387.

16 Kambara T, Simms LA, Whitehall VLJ, et al. BRAF mutation is associated with DNA methylation in serrated polyps and cancers of the colorectum. Gut 2004;53:1137-1144.

17 Nagasaka T, Sasamoto H, Notohara K, et al. Colorectal cancer with mutation in BRAF, KRAS, and wild-type with respect to both oncogenes showing different patterns of DNA methylation. J Clin Oncol 2004;22: 4584-4594.
18 Samowitz W, Albertsen H, Herrick J, et al. Evaluation of a large, population-based sample supports a CpG island methylator phenotype in colon cancer. Gastroenterology 2005;129:837-845.

19 Ogino S, Cantor M, Kawasaki T, et al. CpG island methylator phenotype (CIMP) of colorectal cancer is best characterised by quantitative DNA methylation analysis and prospective cohort studies. Gut 2006; 55:1000-1006.

20 Ogino S, Odze RD, kawasaki T, et al. Correlation of pathologic features with $\mathrm{CpG}$ island methylator phenotype (CIMP) by quantitative DNA methylation analysis in colorectal carcinoma. Am J Surg Pathol 2006;30:1175-1183.

21 Goel A, Li MS, Nagasaka T, et al. Association of JC virus T-antigen expression with the methylator phenotype in sporadic colorectal cancers. Gastroenterology 2006;130:1950-1961.

22 Ogino S, kawasaki T, Kirkner GJ, et al. Down-regulation of p21 (CDKN1A/CIP1) is inversely associated with microsatellite instability and $\mathrm{CpG}$ island methylator phenotype (CIMP) in colorectal cancer. J Pathol 2006;210:147-154.

23 Edmonston TB, Cuesta KH, Burkholder S, et al. Colorectal carcinomas with high microsatellite instability: defining a distinct immunologic and molecular entity with respect to prognostic markers. Hum Pathol 2000;31:1506-1514.

24 Grady WM, Willis JE, Trobridge P, et al. Proliferation and Cdk4 expression in microsatellite unstable colon cancers with TGFBR2 mutations. Int J Cancer 2006; 118:600-608.

25 Eads CA, Danenberg KD, Kawakami K, et al. CpG island hypermethylation in human colorectal tumors is not associated with DNA methyltransferase overexpression. Cancer Res 1999;59:2302-2306.

26 Eads CA, Danenberg KD, Kawakami K, et al. MethyLight: a high-throughput assay to measure DNA methylation. Nucleic Acids Res 2000;28:E32.

27 Widschwendter M, Siegmund KD, Muller HM, et al. Association of breast cancer DNA methylation profiles with hormone receptor status and response to tamoxifen. Cancer Res 2004;64:3807-3813.

28 Ogino S, kawasaki T, Brahmandam M, et al. Precision and performance characteristics of bisulfite conversion and real-time PCR (MethyLight) for quantitative DNA methylation analysis. J Mol Diagn 2006;8:209-217.

29 Colditz GA, Hankinson SE. The Nurses' Health Study: lifestyle and health among women. Nat Rev Cancer 2005;5:388-396.

30 Wei EK, Giovannucci E, Fuchs CS, et al. Low plasma adiponectin levels and risk of colorectal cancer in men: a Prospective Study. J Natl Cancer Inst 2005;97:1688-1694.

31 Ogino S, Brahmandam M, kawasaki T, et al. Combined analysis of COX-2 and p53 expressions reveals synergistic inverse correlations with microsatellite instability and $\mathrm{CpG}$ island methylator phenotype in colorectal cancer. Neoplasia 2006;8:458-464.

32 Ogino S, Brahmandam M, kawasaki T, et al. Epigenetic profiling of synchronous colorectal neoplasias by quantitative DNA methylation analysis. Mod Pathol 2006;19:1083-1090.

33 Ogino S, Kawasaki T, Brahmandam M, et al. Sensitive sequencing method for KRAS mutation detection by Pyrosequencing. J Mol Diagn 2005;7:413-421.

34 Ogino S, Brahmandam $\mathrm{M}$, Cantor $\mathrm{M}$, et al. Distinct molecular features of colorectal carcinoma 
with signet ring cell component and colorectal carcinoma with mucinous component. Mod Pathol 2006; 19:59-68.

35 Eads CA, Lord RV, Wickramasinghe K, et al. Epigenetic patterns in the progression of esophageal adenocarcinoma. Cancer Res 2001;61:3410-3418.

36 Ogino S, kawasaki T, Kirkner GJ, et al. CpG island methylator phenotype-low (CIMP-low) in colorectal cancer: possible associations with male sex and KRAS mutations. J Mol Diagn 2006;8:582-588.

37 Ogino S, Meyerhardt JA, Cantor M, et al. Molecular alterations in tumors and response to combination chemotherapy with gefitinib for advanced colorectal cancer. Clin Cancer Res 2005;11:6650-6656.

38 Muller HM, Millinger S, Fiegl H, et al. Analysis of methylated genes in peritoneal fluids of ovarian cancer patients: a new prognostic tool. Clin Chem 2004;50: 2171-2173.

39 Shen L, Kondo Y, Rosner GL, et al. MGMT promoter methylation and the field defect in sporadic colorectal cancer. J Natl Cancer Inst 2005;97:1330-1338.

40 Giovannucci E, Ogino S. DNA methylation, field effects, and colorectal cancer. J Natl Cancer Inst 2005; 97:1317-1319.
41 Baas IO, Mulder JW, Offerhaus GJ, et al. An evaluation of six antibodies for immunohistochemistry of mutant p53 gene product in archival colorectal neoplasms. J Pathol 1994;172:5-12.

42 Curtin K, Slattery ML, Holubkov $\mathrm{R}$, et al. p53 alterations in colon tumors: a comparison of SSCP/sequencing and immunohistochemistry. Appl Immunohistochem Mol Morphol 2004;12: 380-386.

43 Hall PA, McCluggage WG. Assessing p53 in clinical contexts: unlearned lessons and new perspectives. J Pathol 2006;208:1-6.

44 Palmqvist R, Stenling R, Oberg A, et al. Prognostic significance of p27(Kip1) expression in colorectal cancer: a clinico-pathological characterization. J Pathol 1999;188:18-23.

45 Noguchi T, Kikuchi R, Ono $\mathrm{K}$, et al. Prognostic significance of $\mathrm{p} 27 / \mathrm{kip} 1$ and apoptosis in patients with colorectal carcinoma. Oncol Rep 2003;10: 827-831.

46 Manne U, Jhala NC, Jones J, et al. Prognostic significance of p27(kip-1) expression in colorectal adenocarcinomas is associated with tumor stage. Clin Cancer Res 2004;10:1743-1752.

Supplementary Information accompanies the paper on the Modern Pathology website (http://www.nature. $\mathrm{com} / \operatorname{modpathol)}$ 